\title{
Editorial
}

\section{The new book}

The Internet and digital media are changing science books. They change the way readers approach books and change the way books present their contents. Probably, the Internet and digital media are also changing the contents themselves.

We are witnessing events comparable to the $17^{\text {th }}$ century, when movable type printing created a new market, forever changing the shape of cities and of the territory alike - as skillfully illustrated by Jeremiah Dittmar in his analysis of the correlation between the spreading of printing and the growth of European cities in "Information technology and economic change: The impact of the printing press", published on 11/02/2011 in VOX.

But, above all, movable type printing created new channels to spread culture (in particular in the scientific domain), turning out to be an extraordinary catalyst for the production of new ideas. In short, it marked the birth of the international scientific community. Something similar is probably happening right now with the Internet and digital media, and with the spreading of 'network' relations even outside the web.

Today, Web 2.0 certainly has not changed the way researchers are and operate - also because, at least in some disciplines, they had already been benefiting from the advantages of the net at least from the time of the archives and 'open access' sources. However, Web 2.0 is providing an opportunity to emerge and to enter a network to non-scientists who are positively contaminating scientists with their contribution to the spreading and building of new knowledge in the scientific domain. And at the same time, the Renaissance revolution - the movable type printing - and the modern one - "network" reasoning, action and debate - are crossing their ways, sowing the seed of the birth of the new book.

A few example of these intersections may help to cast light on the new processes now setting off which are to grow bigger and bigger in the next future.

- Google Books is collaborating with publishers, authors and libraries in order to include their books and report book-related information enriched by sentences showing searched terms within their context; in addition, it puts at everyone's disposal books that are out of print or impossible to find.

- Online book sales is a growing market which makes it possible to use any connected computer to buy books that many bookshops are unable to provide equally efficiently.

- Digital printing is now achieving cost and quality levels which are equal to traditional printing or even better then it, as some maintain. This allows publishers to cut manufacturing costs to focus more resources on the quality of books.

- The e-book market is now leaving a phase which has seen a mere translation of texts from paper into PDF format, and from PDF format into e-books, to inaugurate new scenarios in which "books" extend to other contents all around them which contribute to make them multidimension cultural products.

In this framework, we should no longer look at books as physical items, but rather as complex instruments activating a reading process through different steps - among these, physicality, when present, is not necessarily a beginning nor an end, and sometimes not even the main aspect. This new book is therefore particularly versatile and suitable to present and spread scientific knowledge, to make a scientific culture grow, to consolidate an awareness about what science can and cannot do.

The new book - less solemn, austere and distant than its traditional parent - may approach the large groups of people who still do not read quite enough - Italy's figures really are embarrassing to this respect, but even other countries with better results have a wide margin for improvement. Above all, it may help people to act as citizens in a knowledge-oriented society, which cannot happen without including reading as a fundamental and inalienable right-duty. With scientific, medical and technological issues at the heart of the democratic debate and citizens' distance from reading and science being huge, reducing this gap becomes a must - even going beyond the concept of book and extending it to innovative forms which go beyond paper as the sole reading medium. 
Choosing formats extending paper to the web and integrating traditional printing with network production and spreading methods makes it possible to work on many fronts and to talk to different audiences. It makes it possible to shift the focus from print manufacturing processes onto the centrality of the author whom can be given a task putting him/her at the heart of the publishing project, and more generally of culture: the greatest effort should be undertaken to spread the ideas of every single author.

In addition, one should acknowledge that science readers - knowing precisely what they want to read, and willing to overcome many obstacles to find it - make up an ecological niche in the book market. As any other niche, this should be safeguarded by promoting all direct interaction and dialogue forms between author and reader. This can be done more easily through the new book rather than the old one, which was designed according to a top-bottom logic. Besides, these are processes enhancing the chances for exploration and debate on natural phenomena as they encompass dialogue and keep a liquid form open to readers who are actually empowered to build a text: through debate, comments, criticism, dialogue.

Indeed, through web technologies, science books can be made available through time, and dialogue can go alongside books themselves: from blogs through Facebook, passing through Anobii and Twitter, up to traditional media and off-line events. But the actual added value of the network, in all of that, is that it can approach citizens who do not read yet and show them science contents. Many people around us have never bought a book, and see them as high, inaccessible, intellectual, obscure, sometimes hostile: in short, they see them as distant. Books should be brought a click away from all of them, also because many of those people trust science and have great expectations about its role for the development of our societies. Those working in science should then design multi-faceted cultural processes which are to be enticing, so that, when it is the case, the presence of books is well accepted and, after all, may become an option they can at least take into consideration.

Technology, networks, the community spirit have long been strong features of science and may become the guidelines to outline new ways to make people appreciate science readings. The "open access" culture itself offers opportunities not in contrast with market dynamics. Sometimes, they may even fruitfully enhance each other's action.

The methods to achieve all this are yet to come, though some experiments are starting to appear. However, it can be certainly said that the new book, integrated into the web, provided with digital versions and extensions, open to dialogue, debate and experimentation will be one of the knowledge engines. There is an opportunity to set off a new dynamic process having the new book as a flywheel. Science, research, medicine and technology offer the natural contents to get closer to the citizens who will populate our societies in the next few decades.

Translated by Massimo Caregnato

Daniele Gouthier

\section{Notes and references}

${ }^{1}$ http://www.voxeu.org/index.php?q=node/6092.

How TO CITE: $\quad$ D. Gouthier, The new book, Jcom 10(01) (2011) E. 